\title{
Asymptotic Exponential Stability for Diffusion Processes Driven by Stochastic Differential Equations in Duals of Nuclear Spaces
}

\author{
By
}

\author{
Kai LiU* and Tomás CARABAllo**
}

\begin{abstract}
The main objective of this paper is to investigate the asymptotic stability for diffusion processes driven by a class of Itô stochastic differential equations in duals of nuclear spaces. A coercivity condition imposed on this sort of equation plays the role of an exponential stability criterion. An example is studied to illustrate our theory.
\end{abstract}

\section{$\S 1$. Introduction}

In the paper we shall study the exponential stability of stochastic diffusion equations in duals of nuclear spaces. These equations naturally arise in the research of chemical reaction-diffusion equations, neurophysiology and turbulence, especially, in the recent river pollution model researches (see [11], [15] and [16]). Roughly speaking, we shall consider the following stochastic diffusion equation:

$$
X_{t}=X_{0}+\int_{0}^{t} A\left(s, X_{s}\right) d s+\int_{0}^{t} B\left(s, X_{s}\right) d W_{s}
$$

where $A: \mathbf{R}_{+} \times \Phi^{\prime} \rightarrow \Phi^{\prime}, B: \mathbf{R}_{+} \times \Phi^{\prime} \rightarrow \mathcal{L}\left(\Phi^{\prime}, \Phi^{\prime}\right)$ are two measurable mappings and $W_{t}$ is a $\Phi^{\prime}$-valued Wiener process. Here $\Phi^{\prime}$ is the dual space of a certain

Communicated by Y. Takahashi, June 19, 2000. Revised September 25, 2000.

2000 Mathematics Subject Classification(s): Primary, 93E03; Secondary 60H10.

* Department of Probability and Statistics, The University of Sheffield, The Hicks Building, Hounsfield Road, Sheffield, S3 7RH, United Kingdom.

e-mail: k.liu@sheffield.ac.uk

** Dpto. Ecuaciones Diferenciales y Análisis Numérico, Universidad de Sevilla. Apartado de Correos 1160, 41080-Sevilla, SPAIN.

e-mail: caraball@cica.es 
countably Hilbertian nuclear space and $\mathcal{L}\left(\Phi^{\prime}, \Phi^{\prime}\right)$ is the space of all bounded linear operators from $\Phi^{\prime}$ into itself.

Diffusion equations of the type (1.1) have been studied by a number of authors, for instance, G. Kallianpur and R. L. Wolpert [10], G. Kallianpur and J. Xiong [11], H. Tuckwell [15] and J. B. Walsh [16] among others. The reader is referred to G. Kallianpur and J. Xiong [11] for further details concerning certain properties of the solutions (1.1) and some related topics. In the paper, we are particularly interested in the criteria of exponential stability in the sense of mean square and pathwise with probability one of the strong solutions to the equations (1.1).

It is a long history for the investigation of the exponential stability of stochastic differential equations in finite dimensional spaces and, more recently, of stochastic evolution equations in Hilbert spaces. For infinite dimensional cases, we should mention U. G. Haussmann [6] (linear case) and A. Ichikawa [7] (semilinear case) for their fundamental work on this aspect. Nevertheless, for nuclear space-valued stochastic differential equation situations, to the best of our knowledge it seems that nobody ever carried out the study of exponential stability either in the sense of mean square or pathwise with probability one. This is the main task in this paper to fill this gap. It is particularly worth pointing out that our approaches, which are devoted to the consideration of the stochastic differential equations in duals of nuclear spaces (1.1), could even be used to extend the results of [6], [7] to cover general non-autonomous Hilbert space-valued stochastic differential systems. Firstly, we shall give sufficient conditions for the exponential stability in mean square of the strong solutions to the equations (1.1). Next, we obtain exponential stability of paths with probability one. Our argument is based on a coercivity condition which plays a key role for the existence and uniqueness of the equations (1.1). As a consequence, we will observe how a suitable coercivity condition may be regarded as an exponential stability criterion.

The exposition is as follows. In Section 2, we shall briefly collect some notions and notations which are essential for our stability analysis. Section 3 is devoted to the investigation of exponentially asymptotic stability of strong solutions. Finally, in Section 4 we will illustrate the theorems derived in the last section by studying an example.

\section{§2. Preliminaries}

In this section we are going to state some basic notions and notations in a suitable way. In particular, the reader is strongly referred to G. Kallianpur and 
J. Xiong [11] for a systematic and detailed statement concerning the material in this section.

Let $\Phi$ be a separable Fréchet space which is a countably Hilbertian space, that is, its topology is given by an increasing sequence $\|\cdot\|_{n}, n \geq 0$, of compatible Hilbertian norms. In particular, throughout this paper we suppose $\Phi$ is nuclear, precisely, for each $n \geq 0$ there exists $m>n$ such that the canonical injection from $\Phi_{m}$ into $\Phi_{n}$ is Hilbert-Schmidt. Here $\Phi_{n}$ is the completion of $\Phi$ with respect to $\|\cdot\|_{n}$. Let $\Phi^{\prime}$ be the collection of all continuous linear maps from $\Phi$ to $\mathbf{R}$, i.e., the dual space of $\Phi$. We could show that $\left\{\Phi_{n}\right\}_{n \geq 0}$ is a sequence of decreasing Hilbertian spaces and $\Phi=\cap_{n=0}^{\infty} \Phi_{n}$. Identifying $\Phi_{0}^{\prime}$ with $\Phi_{0}$ by Riesz's representation theorem, we denote $\Phi_{n}^{\prime}$ by $\Phi_{-n}$ with norms $\|\cdot\|_{-n}, n \geq 0$. Then $\left\{\Phi_{-n}\right\}_{n \geq 0}$ is a sequence of increasing Hilbertian spaces, $\Phi^{\prime}$ is sequentially complete and $\Phi^{\prime}=\cup_{n=0}^{\infty} \Phi_{-n}$. In the latter case, we shall denote by $\left\{\phi_{j}^{p}\right\} \subset \Phi$ a complete orthonormal system, or simply, CONS of $\Phi_{p}$ and $\left\{\phi_{j}^{-p}\right\}$ the CONS of $\Phi_{-p}$ conjugate to $\left\{\phi_{j}^{p}\right\}$ for $p \geq 0$. Let $\theta_{p}$ be the isometry from $\Phi_{-p}$ to $\Phi_{p}$ such that $\theta_{p} \phi_{j}^{-p}=\phi_{j}^{p}, \forall j \geq 1$.

A class of important examples of countably Hilbertian spaces can be described appropriately as follows. Let $H$ be a real separable Hilbert space with inner product $\langle\cdot, \cdot\rangle_{H}$, and $A=-L$ a closed densely defined self-adjoint operator on $H$ such that $\langle-L \phi, \phi\rangle_{H} \leq 0$ for $\phi \in \operatorname{Dom}(L)$, the domain of $L$. Let $\left\{T_{t}\right\}$ be the semigroups on $H$ determined by $A$. Further assume that some power of the resolvent of $L$ is a Hilbert-Schmidt operator, i.e.,

$$
\exists r_{1} \text { such that }(\lambda I+L)^{-r_{1}} \text { is Hilbert-Schmidt. }
$$

This condition enables us to prove that there exist $0 \leq \lambda_{1} \leq \lambda_{2} \leq \cdots$ and $\left\{\phi_{j}\right\} \subset H$, a CONS of $H$, such that

$$
L \phi_{j}=\lambda_{j} \phi_{j}, \quad \text { for any } \quad j \geq 1 .
$$

Define

$$
\begin{aligned}
\Phi & =\left\{\phi \in H:\left\|(I+L)^{r} \phi\right\|_{H}^{2}<\infty, \quad \forall r \in \mathbf{R}\right\} \\
& =\left\{\phi \in H: \sum_{j=1}^{\infty}\left(1+\lambda_{j}\right)^{2 r}\left\langle\phi, \phi_{j}\right\rangle_{H}^{2}<\infty, \quad \forall r \in \mathbf{R}\right\}
\end{aligned}
$$

and the inner product $\langle\cdot, \cdot\rangle_{r}$ on $\Phi$ by

$$
\langle\phi, \psi\rangle_{r}=\sum_{j=1}^{\infty}\left(1+\lambda_{j}\right)^{2 r}\left\langle\phi, \phi_{j}\right\rangle_{H}\left\langle\psi, \phi_{j}\right\rangle_{H}
$$


and

$$
\|\phi\|_{r}^{2}=\langle\phi, \phi\rangle_{r}
$$

Let $\Phi_{r}$ be the $\|\cdot\|_{r}$-completion of $\Phi$. We then have

$$
\Phi=\bigcap_{r} \Phi_{r}, \quad \Phi^{\prime}=\bigcup_{r} \Phi_{r}
$$

and for $r \leq s, \phi \in \Phi,\|\phi\|_{r} \leq\|\phi\|_{s}$ and furthermore $\Phi_{s} \subset \Phi_{r}$ with $\Phi_{0}=H$. Condition (2.1) implies that the injection from $\Phi_{q}$ into $\Phi_{p}$ is Hilbert-Schmidt for $q \geq p+r_{1}$ and therefore $\Phi$ is a countably Hilbertian nuclear space, simply, CHNS. As usual, we also call the compatible family $\left(\Phi, H, T_{t}\right)$ or $(\Phi, H, L)$ a special compatible family.

We assume throughout that $\left(\Omega, \mathcal{F},\left\{\mathcal{F}_{t}\right\}_{t \geq 0}, P\right)$ is a complete probability space with a right continuous filtration $\left\{\mathcal{F}_{t}\right\}_{t \geq 0}$. A map $X: \Omega \rightarrow \Phi^{\prime}$ is a $\Phi^{\prime}-$ valued random variable if it is $\mathcal{F} / \mathcal{B}\left(\Phi^{\prime}\right)$-measurable, where $\mathcal{B}\left(\Phi^{\prime}\right)$ is the Borel field of the topological space $\Phi^{\prime}$ (in the sense of strong topology). A family $\left\{X_{t} ; t \in \mathbf{R}_{+}\right\}$of $\Phi^{\prime}$-valued random variables is called a $\Phi^{\prime}$-process.

In the rest of this paper, we shall concern with $\Phi^{\prime}$-valued martingales. In particular, we have the following:

Definition 2.1. A $\Phi^{\prime}$-valued process $M=\left\{M_{t}\right\}_{t \geq 0}$ is a $\Phi^{\prime}$-martingale with respect to $\left\{\mathcal{F}_{t}\right\}_{t \geq 0}$ if for each $\phi \in \Phi, M_{t}[\phi]$ is a martingale with respect to $\left\{\mathcal{F}_{t}\right\}$. It is called a $\Phi^{\prime}$-square-integrable-martingale if, in addition,

$$
E\left(M_{t}[\phi]^{2}\right)<\infty, \quad \forall \phi \in \Phi, \quad t \geq 0 .
$$

We let $\mathcal{M}\left(\Phi^{\prime}\right)$ (resp. $\mathcal{M}^{2}\left(\Phi^{\prime}\right)$ ) denote the collection of all $\Phi^{\prime}$-martingales (resp. $\Phi^{\prime}$-square-integrable-martingales). We also let $\mathcal{M}^{2, c}\left(\Phi^{\prime}\right)=\left\{M \in \mathcal{M}^{2}\left(\Phi^{\prime}\right): M_{t}[\phi]\right.$ has a continuous version for each $\left.\phi \in \Phi\right\}$.

Definition 2.2. A continuous (in the sense of strong topology) $\Phi^{\prime}$ valued stochastic process $W=\left(W_{t}\right)_{t \geq 0}$ on $(\Omega, \mathcal{F}, P)$ is called a centered $\Phi^{\prime}$ Wiener process with $Q(\cdot, \cdot)$ if $W$ satisfies the following three conditions:

a) $W_{0}=0$ a.s.;

b) $W$ has independent increments, i.e., the random variables

$$
W_{t_{1}}\left[\phi_{1}\right], \quad\left(W_{t_{2}}-W_{t_{1}}\right)\left[\phi_{2}\right], \ldots,\left(W_{t_{n}}-W_{t_{n-1}}\right)\left[\phi_{n}\right]
$$

are independent for any $\phi_{1}, \phi_{2}, \ldots, \phi_{n} \in \Phi, 0 \leq t_{1} \leq \cdots \leq t_{n}, n \geq 1$; 
c) For each $t \geq 0$ and $\phi \in \Phi$

$$
E\left(e^{i W_{t}[\phi]}\right)=e^{-t Q(\phi, \phi) / 2}
$$

where $Q$ is a covariance functional, i.e., a positive definite symmetric continuous bilinear form on $\Phi \times \Phi$.

Clearly, $W \in \mathcal{M}^{2, c}\left(\Phi^{\prime}\right),\left\{W_{t}[\phi]: \phi \in \Phi, \quad t \geq 0\right\}$ is a centered Gaussian system and

$$
E\left(W_{t}[\psi] W_{s}[\phi]\right)=(s \wedge t) Q(\psi, \phi), \quad \psi, \phi \in \Phi, \quad s, t \geq 0 .
$$

Definition 2.3. Let $H$ be a separable Hilbert space with norm $\|\cdot\|_{H}$. A family $\left\{B_{t}(h): t \geq 0, \quad h \in H\right\}$ of real-valued random variables is called a cyclindrical Brownian motion (c.B.m) on $H$ with covariance $\Sigma$ if $\Sigma$ is a continuous self-adjoint positive definite operator on $H$ such that the following conditions hold:

i) For each $h \in H$ such that $h \neq 0,\langle\Sigma h, h\rangle_{H}^{-1 / 2} B_{t}(h)$ is a one-dimensional standard Wiener process;

ii) For each $t \geq 0, \alpha_{1}, \alpha_{2} \in \mathbf{R}$ and $f_{1}, f_{2} \in H$

$$
B_{t}\left(\alpha_{1} f_{1}+\alpha_{2} f_{2}\right)=\alpha_{1} B_{t}\left(f_{1}\right)+\alpha_{2} B_{t}\left(f_{2}\right) \quad \text { a.s.; }
$$

iii) For each $h \in H,\left\{B_{t}(h)\right\}$ is an $\mathcal{F}_{t}^{B}$-martingale, where

$$
\mathcal{F}_{t}^{B}=\sigma\left\{B_{s}(h): s \leq t, \quad h \in H\right\} .
$$

$\left\{B_{t}(h): t \geq 0, \quad h \in H\right\}$ is called a standard $H$-c.B.m or simply, $H$-c.B.m. if it is a $H$-c.B.m. with covariance $\Sigma=I$.

For each $\phi \in \Phi$, let $\imath \phi:=Q(\phi, \cdot)$. Then $\imath$ is an injective linear operator from $\Phi$ onto a linear subspace $\mathcal{R}(\imath)$ of $\Phi^{\prime}$. In particular, for arbitrary $v_{1}, v_{2} \in \mathcal{R}(\imath)$, let $\left\langle v_{1}, v_{2}\right\rangle_{H_{Q}}:=Q\left(\imath^{-1} v_{1}, \imath^{-1} v_{2}\right)$. Then $\langle\cdot, \cdot\rangle_{H_{Q}}$ is an inner product on $\mathcal{R}(\imath)$. Let $\|\cdot\|_{H_{Q}}$ be the norm on $\mathcal{R}(\imath)$ determined by the inner product $\langle\cdot, \cdot\rangle_{H_{Q}}$ and let $H_{Q}$ be the completion of $\mathcal{R}(\imath)$ with respect to $\|\cdot\|_{H_{Q}}$. Then $H_{Q}$ is a separable Hilbert space and $H_{Q} \subset \Phi^{\prime}$. It could also be shown that there exists a one-toone correspondence between a $\Phi^{\prime}$-valued Wiener process $W$ with covariance $Q$ and an $H_{Q^{-}}$-B.B.m. $B$ :

$$
W_{t}=\sum_{j=1}^{\infty} B_{t}\left(f_{j}\right) f_{j}
$$

where $\left\{f_{j}\right\}$ is a CONS of $H_{Q}$;

$$
B_{t}(v)=\lim _{n \rightarrow \infty} W_{t}\left[\imath^{-1} v_{n}\right], \quad \forall v \in H_{Q}
$$


where $\left\{v_{n}\right\} \subset \mathcal{R}(\imath)$ converges to $v$ in $H_{Q}$.

Consider the following stochastic diffusion equation (see [11] for further details on stochastic integral and related properties)

$$
X_{t}=X_{0}+\int_{0}^{t} A\left(s, X_{s}\right) d s+\int_{0}^{t} B\left(s, X_{s}\right) d W_{s}
$$

where $A: \mathbf{R}_{+} \times \Phi^{\prime} \rightarrow \Phi^{\prime}, B: \mathbf{R}_{+} \times \Phi^{\prime} \rightarrow \mathcal{L}\left(\Phi^{\prime}, \Phi^{\prime}\right)$ are two measurable mappings and $W_{t}$ is a $\Phi^{\prime}$-valued Wiener process. Here $\mathcal{L}\left(\Phi^{\prime}, \Phi^{\prime}\right)$ denotes the collection of all continuous linear mappings from $\Phi^{\prime}$ into $\Phi^{\prime}$.

Definition 2.4. Let $\left(\Omega, \mathcal{F},\left\{\mathcal{F}_{t}\right\}_{t \geq 0}, P\right)$ be the stochastic basis and $W_{t}$ a $\Phi^{\prime}$-valued Wiener process with covariance function $Q$. Suppose that $X_{0}$ is a $\Phi_{-p}$-valued random variable such that $E\left\|X_{0}\right\|_{-p}^{2}<\infty$. Then by a $\Phi_{-p}$-valued strong solution on $\Omega$ to the $\operatorname{SDE}(2.8)$ for $t \in[0, T]$ we mean a process $X_{t}$ defined on $\Omega$ such that

(a) $X_{t}$ is a $\Phi_{-p}$-valued $\mathcal{F}_{t}$-measurable random variable;

(b) $X_{t} \in C\left([0, T], \Phi_{-p}\right)$, a.s.;

(c) There exists a sequence $\left(\sigma_{n}\right)$ of bounded stopping times on $\Omega$ increasing to infinity such that $\forall n \geq 1$

$$
E \int_{0}^{T \wedge \sigma_{n}}\left\|A\left(s, X_{s}\right)\right\|_{-q} d s<\infty
$$

and

$$
E \int_{0}^{T \wedge \sigma_{n}}\left\|B\left(s, X_{s}\right)\right\|_{L_{(2)}\left(H_{Q}, \Phi_{-p}\right)}^{2} d s<\infty .
$$

Here $L_{(2)}\left(H_{Q}, \Phi_{-p}\right)$ denotes the class of all Hilbert-Schmidt operators from $H_{Q}$ into $\Phi_{-p}$ and $q$ will be introduced in the following assumption (H1);

(d) The $\operatorname{SDE}(2.8)$ is satisfied for all $t \in[0, T]$ and almost all $\omega \in \Omega$.

If $T$ is replaced by $\infty$, we call $X_{t}$ a global strong solution of (2.8).

As we are mainly interested in the stability analysis, one always assumes that the equation (2.8) has a unique global strong solution. In particular, for this purpose we shall make the following assumption (H1) [11]:

There exists an index $p_{0}>0$ such that, $\forall p \geq p_{0}, \exists q \geq p$ and a constant $K=K(p, q)>0$ such that

(D1) (Continuity) $\forall t \in \mathbf{R}_{+}$, the maps $v \in \Phi_{-p} \rightarrow A(t, v) \in \Phi_{-q}$ and $v \in \Phi_{-p} \rightarrow B(t, v) \in L_{(2)}\left(H_{Q}, \Phi_{-p}\right)$ are continuous;

(D2) (Coercivity) $\forall t \in \mathbf{R}_{+}$and $v \in \Phi_{-p}$, we have

$$
2 A(t, v)\left[\theta_{p} v\right]+\|B(t, v)\|_{L_{(2)}\left(H_{Q}, \Phi_{-p}\right)}^{2} \leq K\left(1+\|v\|_{-p}^{2}\right) ;
$$


(D3) (Growth) $\forall t \in \mathbf{R}_{+}$and $v \in \Phi_{-p}$, we have

$$
\|A(t, v)\|_{-q}^{2} \leq K\left(1+\|v\|_{-p}^{2}\right) ;
$$

(D4) (Lipschitz) $\forall t \in \mathbf{R}_{+}, v_{1}, v_{2} \in \Phi_{-p}$, we have

$$
\left\|A\left(t, v_{1}\right)-A\left(t, v_{2}\right)\right\|_{-q} \leq K\left\|v_{1}-v_{2}\right\|_{-p}
$$

and

$$
\left\|B\left(t, v_{1}\right)-B\left(t, v_{2}\right)\right\|_{L_{(2)}\left(H_{Q}, \Phi_{-p}\right)} \leq K\left\|v_{1}-v_{2}\right\|_{-p} .
$$

\section{§3. Main Results}

In this section, we shall devote ourselves to the investigation of exponential stability of the equation (2.8). For simplicity, throughout this section we take the special compatible family $(\Phi, H, L)$ described in Section 1 as our basic CHNS. In particular, to our end we shall make the following additional assumption (H2):

$\forall t \in \mathbf{R}_{+}, v \in \Phi_{-p}, p \geq p_{0}$, there exist positive constants $\nu>0, \mu>0$, $p \leq r \leq q$ and positive function $\gamma(t), t \in \mathbf{R}_{+}$, such that

$$
2 A(t, v)\left[\theta_{q} v\right]+\|B(t, v)\|_{L_{(2)}\left(H_{Q}, \Phi_{-r}\right)}^{2} \leq-\nu\|v\|_{-r}^{2}+\gamma(t) e^{-\mu t}
$$

where $p_{0}, q$ are introduced as in the assumption $(\mathbf{H 1})$ and $\gamma(t)$ satisfies that for arbitrary $\delta>0, \gamma(t)=o\left(e^{\delta t}\right)$, as $t \rightarrow \infty$, i.e., $\lim _{t \rightarrow \infty} \gamma(t) / e^{\delta t}=0$.

Before proceeding to our stability arguments, let us first make the following comments on the condition (H2):

Remark 1. As is well known, the coercivity condition (2.11) plays an essential role in the establishment of the existence and uniqueness of the equation (2.8). The further restrictive coercivity condition (3.1) will play the role of an exponential stability criterion as described below.

Remark 2. The exponential decay term appearing on the right hand side of (3.1) is of the essence for our stability purposes. In fact, to see this, let us simply consider the following one dimensional linear Itô equation:

Example 3.1. Assume $X_{t}$ satisfies the following

$$
d X_{t}=-p X_{t} d t+(1+t)^{-q} d W_{t}, \quad t \geq 0
$$


with initial data $X_{0}=0$, where $p, q>0$ are two positive constants and $W_{t}$ is a one-dimensional standard Brownian motion.

Clearly, the left-hand side of the coercivity type condition (3.1) now turns out to be

$$
2\langle-p v, v\rangle+\left[(1+t)^{-q}\right]^{2}=-2 p v^{2}+(1+t)^{-2 q} .
$$

where $\langle\cdot, \cdot\rangle$ denotes the standard inner product in $\mathbf{R}$. However, since the last term $(1+t)^{-2 q}$ is not exponentially decreasing, the solution is exponentially unstable. Indeed, it is easy to obtain the explicit solution

$$
X_{t}=e^{-p t} \int_{0}^{t} e^{p s} \cdot(1+s)^{-q} d W_{s}=: e^{-p t} M_{t}, \quad t \geq 0,
$$

which immediately implies that for arbitrarily given $q>0$ Lyapunov exponent

$$
\lim _{t \rightarrow \infty} \frac{\log E\left|X_{t}\right|^{2}}{t}=0 .
$$

In the meantime, noticing the law of the iterated logarithm

$$
\limsup _{t \rightarrow \infty} \frac{M_{t}}{\sqrt{2\left\langle M_{t}\right\rangle \log \log \left\langle M_{t}\right\rangle}}=1 \quad \text { a.s. }
$$

and

$$
\limsup _{t \rightarrow \infty} \frac{\log \left(\int_{0}^{t} e^{2 p s}(1+s)^{-2 q} d s\right)}{t}=2 p,
$$

we therefore get Lyapunov exponent

$$
\limsup _{t \rightarrow \infty} \frac{1}{t} \log \left|X_{t}\right|=0 \quad \text { a.s. }
$$

That is, in spite of the typical stability of an ordinary differential equation

$$
d X_{t}=-p X_{t} d t
$$

the polynomial type decay of the noise term is not sufficient to ensure the exponential stability of its stochastically perturbed system.

Now we are in a position to obtain our main results in the paper.

Theorem 3.2. $\quad$ Suppose $X_{t}$ is a solution to the equation (2.8) satisfying (H1). Furthermore we assume the coercivity condition (3.1) holds. Then there exist constants $\tau>0, C>0$ such that

$$
E\left\|X_{t}\right\|_{-r}^{2} \leq C \cdot e^{-\tau t}, \quad \forall t \geq 0 .
$$

That is, the strong solution is exponentially stable in mean square. In particular, constant $\tau>0$ can be taken as follows: $\tau<\mu$, if $\mu \leq \nu$ and $\tau=\nu$, if $\mu>\nu$. 
Proof. For arbitrary $\phi \in \Phi$, we have

$$
\begin{aligned}
X_{t}[\phi]= & \int_{0}^{t} A\left(s, X_{s}\right)[\phi] d s \\
& +\sum_{j} \int_{0}^{t}\left\langle B\left(s, X_{s}\right)^{\prime} \phi, v_{j}\right\rangle_{H_{Q}} d W_{s}\left[l^{-1} v_{j}\right],
\end{aligned}
$$

where $\left\{v_{j}\right\} \subset \mathcal{R}(\imath)$ is a CONS of $H_{Q}$ and $\imath$ is defined as in Section 2. Here $B(s, \cdot)^{\prime}$ denotes the dual operator of $B(s, \cdot) \in \mathcal{L}\left(H_{Q}, \Phi_{-r}\right), s \geq 0$. It follows from Itô's formula and Definition 2.4 that for arbitrary $\delta>0$ with $\mu-\delta>0$, we have

$$
\begin{aligned}
& e^{(\mu-\delta) t \wedge \sigma_{n}} X_{t \wedge \sigma_{n}}[\phi]^{2}-X_{0}[\phi]^{2} \\
& =(\mu-\delta) \int_{0}^{t \wedge \sigma_{n}} e^{(\mu-\delta) s} X_{s}[\phi]^{2} d s+2 \int_{0}^{t \wedge \sigma_{n}} e^{(\mu-\delta) s} X_{s}[\phi] A\left(s, X_{s}\right)[\phi] d s \\
& \quad+2 \sum_{j} \int_{0}^{t \wedge \sigma_{n}} e^{(\mu-\delta) s} X_{s}[\phi]\left\langle B\left(s, X_{s}\right)^{\prime} \phi, v_{j}\right\rangle_{H_{Q}} d W_{s}\left[l^{-1} v_{j}\right] \\
& \quad+\int_{0}^{t \wedge \sigma_{n}} e^{(\mu-\delta) s} Q\left(B\left(s, X_{s}\right)^{\prime} \phi, B\left(s, X_{s}\right)^{\prime} \phi\right) d s
\end{aligned}
$$

where $\left(\sigma_{n}\right)$ is the sequence of stopping times defined as in Definition 2.4. Now, since $\int_{0}^{t \wedge \sigma_{n}} e^{(\mu-\delta) s} X_{s}[\phi]\left\langle B\left(s, X_{s}\right)^{\prime} \phi, v_{j}\right\rangle_{H_{Q}} d W_{t}\left[l^{-1} v_{j}\right], t \in \mathbf{R}_{+}$, is a continuous martingale, it follows that

$$
E\left(\int_{0}^{t \wedge \sigma_{n}} e^{(\mu-\delta) s} X_{s}[\phi]\left\langle B\left(s, X_{s}\right)^{\prime} \phi, v_{j}\right\rangle_{H_{Q}} d W_{s}\left[\imath^{-1} v_{j}\right]\right)=0, \quad t \in \mathbf{R}_{+} .
$$

Therefore, letting $\phi=\phi_{k}^{r}, n \rightarrow \infty, k \in \mathbf{N}$ and then adding on index $k \in \mathbf{N}$, we can deduce by Fatou's lemma and the condition (3.1)

$$
\begin{aligned}
& E e^{(\mu-\delta) t}\left\|X_{t}\right\|_{-r}^{2} \\
& \quad \leq E\left\|X_{0}\right\|_{-r}^{2}+(\mu-\delta-\nu) \int_{0}^{t} e^{(\mu-\delta) s} E\left\|X_{s}\right\|_{-r}^{2} d s+\int_{0}^{t} \gamma(s) e^{-\delta s} d s
\end{aligned}
$$

If $\mu-\nu \leq 0$, we therefore deduce

$$
E e^{(\mu-\delta) t}\left\|X_{t}\right\|_{-r}^{2} \leq E\left\|X_{0}\right\|_{-r}^{2}+\int_{0}^{t} \gamma(s) e^{-\delta s} d s,
$$

that is, letting $k(\delta)=\int_{0}^{\infty} \gamma(s) e^{-\delta s} d s$, we have

$$
E\left\|X_{t}\right\|_{-r}^{2} \leq\left(E\left\|X_{0}\right\|_{-r}^{2}+k(\delta)\right) e^{-(\mu-\delta) t} .
$$


On the other hand, if $\mu-\nu>0$, it is always possible to choose a suitable $\delta>0$ such that $\mu-\nu-\delta>0$. Then, by virtue of Gronwall's lemma we easily derive from (3.5) that

$$
E e^{(\mu-\delta) t}\left\|X_{t}\right\|_{-r}^{2} \leq\left(E\left\|X_{0}\right\|_{-r}^{2}+\int_{0}^{t} \gamma(s) e^{-\delta s} d s\right) e^{(\mu-\delta-\nu) t} .
$$

Hence, letting $\delta>0$ small enough immediately yields that there exists a constant $k(\delta)>0$ such that

$$
E\left\|X_{t}\right\|_{-r}^{2} \leq\left(E\left\|X_{0}\right\|_{-r}^{2}+k(\delta)\right) e^{-\nu t}
$$

Combining the arguments above, we thus obtain our conclusion.

Theorem 3.3. $\quad$ Assume the assumptions in Theorem 3.2 hold. Then there exist positive constants $M, \beta$ and a subset $\Omega_{0} \subset \Omega$ with $P\left(\Omega_{0}\right)=0$ such that, for each $\omega \notin \Omega_{0}$, there exists a positive random number $T(\omega)$ such that the following holds:

$$
\left\|X_{t}\right\|_{-r}^{2} \leq M \cdot e^{-\beta t}, \quad \forall t \geq T(\omega) .
$$

That is, the strong solution is almost surely stable.

Proof. Our proofs are divided into the following several steps.

Step 1 . We firstly claim that there exists a constant $C>0$, independent of $t \in \mathbf{R}_{+}$, such that

$$
\int_{s}^{t} E\left\|B\left(u, X_{u}\right)\right\|_{L_{(2)}\left(H_{Q}, \Phi_{-r}\right)}^{2} d u \leq C<\infty, \quad 0 \leq s \leq t .
$$

Indeed, applying Itô's formula as in Theorem 3.2 to (2.8), we can get (3.5) once more. If $\mu-\nu \leq 0$, we therefore deduce

$$
\int_{0}^{t} e^{(\mu-\delta) s} E\left\|X_{s}\right\|_{-r}^{2} d s \leq \frac{E\left\|X_{0}\right\|_{-r}^{2}+\int_{0}^{t} \gamma(s) e^{-\delta s} d s}{\nu+\delta-\mu} .
$$

On the other hand, letting $v=0$ in (3.1) yields that for any $t \geq 0$,

$$
\|B(t, 0)\|_{L_{(2)}\left(H_{Q}, \Phi_{-r}\right)}^{2} \leq \gamma(t) e^{-\mu t},
$$

which, together with (2.14) and (3.8), immediately implies

$$
\int_{0}^{t} e^{(\mu-\delta) s} E\left\|B\left(s, X_{s}\right)\right\|_{L_{(2)}\left(H_{Q}, \Phi_{-r}\right)}^{2} d s
$$




$$
\begin{aligned}
& \leq 2 \int_{0}^{t} e^{(\mu-\delta) s} E\|B(s, 0)\|_{L_{(2)}\left(H_{Q}, \Phi_{-r}\right)}^{2} d s+2 K^{2} \int_{0}^{t} e^{(\mu-\delta) s} E\left\|X_{s}\right\|_{-r}^{2} d s \\
& \leq 2 \int_{0}^{\infty} \gamma(s) e^{-\delta s} d s+\frac{2 K^{2}}{\nu+\delta-\mu}\left(E\left\|X_{0}\right\|_{-r}^{2}+\int_{0}^{\infty} \gamma(s) e^{-\delta s} d s\right) \\
& =: C(\delta),
\end{aligned}
$$

and, for $0 \leq s \leq t$, we get

$$
\int_{s}^{t} e^{(\mu-\delta) u} E\left\|B\left(u, X_{u}\right)\right\|_{L_{(2)}\left(H_{Q}, \Phi_{-r}\right)}^{2} d u \leq C(\delta),
$$

and

$$
e^{-(\mu-\delta) s} \int_{s}^{t} e^{(\mu-\delta) u} E\left\|B\left(u, X_{u}\right)\right\|_{L_{(2)}\left(H_{Q}, \Phi_{-r}\right)}^{2} d u \leq C(\delta) \cdot e^{-(\mu-\delta) s}, \quad 0 \leq s \leq t .
$$

Therefore,

$$
\begin{aligned}
& \int_{s}^{t} E\left\|B\left(u, X_{u}\right)\right\|_{L_{(2)}\left(H_{Q}, \Phi_{-r}\right)}^{2} d u \\
& \quad \leq \int_{s}^{t} e^{(\mu-\delta)(u-s)} E\left\|B\left(u, X_{u}\right)\right\|_{L_{(2)}\left(H_{Q}, \Phi_{-r}\right)}^{2} d u \\
& \quad \leq C(\delta) \cdot e^{-(\mu-\delta) s}, \quad 0 \leq s \leq t .
\end{aligned}
$$

On the other hand, if $\mu-\nu>0$, it is always possible to choose a suitable $\delta>0$ such that $\mu-\nu-\delta>0$. Then, by virtue of Gronwall's lemma we easily deduce from (3.5) that

$$
E e^{(\mu-\delta) t}\left\|X_{t}\right\|_{-r}^{2} \leq\left(E\left\|X_{0}\right\|_{-r}^{2}+\int_{0}^{t} \gamma(s) e^{-\delta s} d s\right) e^{(\mu-\delta-\nu) t} .
$$

Hence, letting $\delta>0$ small enough immediately yields that

$$
\int_{0}^{t} e^{(\nu-\delta) s} E\left\|X_{s}\right\|_{-r}^{2} d s \leq\left(E\left\|X_{0}\right\|_{-r}^{2}+\int_{0}^{t} \gamma(s) e^{-\delta s} d s\right) \int_{0}^{t} e^{-\delta s} d s .
$$

A similar argument as above once more yields that there exists a $C(\delta)>0$ such that

$$
\int_{s}^{t} E\left\|B\left(u, X_{u}\right)\right\|_{L_{(2)}\left(H_{Q}, \Phi_{-r}\right)}^{2} d u \leq C(\delta) \cdot e^{-(\nu-\delta) s}, \quad 0 \leq s \leq t,
$$

and hence our claim is proved.

Step 2. Next we claim that there exists a positive constant $M>0$ such that

$$
E\left(\sup _{0 \leq t<T}\left\|X_{t}\right\|_{-r}^{2}\right) \leq M
$$


for any $T>0$. Indeed, applying Itô's formula and Fatou's lemma to (3.4) yields that for arbitrary $\phi \in \Phi$,

$$
\begin{aligned}
X_{t}[\phi]^{2}- & X_{0}[\phi]^{2} \\
\leq & 2 \int_{0}^{t} X_{s}[\phi] A\left(s, X_{s}\right)[\phi] d s+\int_{0}^{t} Q\left(B\left(s, X_{s}\right)^{\prime} \phi, B\left(s, X_{s}\right)^{\prime} \phi\right) d s \\
& +2 \sum_{j} \int_{0}^{t} X_{s}[\phi]\left\langle B\left(s, X_{s}\right)^{\prime} \phi, v_{j}\right\rangle_{H_{Q}} d W_{s}\left[\imath^{-1} v_{j}\right]
\end{aligned}
$$

where $\left\{v_{j}\right\} \subset \mathcal{R}(\imath)$ is a CONS of $H_{Q}$ and $\imath$ is defined as above.

On the other hand, by virtue of Burkholder-Davis-Gundy's inequality, for any $T \in \mathbf{R}_{+}$we obtain

$$
\begin{aligned}
E\left[\sup _{t \in[0, T]}\left|\sum_{j} \int_{0}^{t} X_{s}[\phi]\left\langle B\left(s, X_{s}\right)^{\prime} \phi, v_{j}\right\rangle_{H_{Q}} d W_{s}\left[\imath^{-1} v_{j}\right]\right|\right] \\
\leq C_{1} E\left[\left(\sum_{j} \int_{0}^{T}\left|X_{s}[\phi]\right|^{2}\left\langle B\left(s, X_{s}\right)^{\prime} \phi, v_{j}\right\rangle_{H_{Q}}^{2} d s\right)^{\frac{1}{2}}\right] \\
\leq C_{1} E\left\{\sup _{0 \leq s \leq T}\left|X_{s}[\phi]\right|\left[\int_{0}^{T}\left\|B\left(s, X_{s}\right)^{\prime} \phi\right\|_{H_{Q}}^{2} d s\right]^{\frac{1}{2}}\right\} \\
\leq \frac{1}{2} E\left(\sup _{0 \leq s \leq T}\left|X_{s}[\phi]\right|^{2}\right)+C_{2} \int_{0}^{T} E\left\|B\left(s, X_{s}\right)^{\prime} \phi\right\|_{H_{Q}}^{2} d s
\end{aligned}
$$

where $C_{1}, C_{2}$ are two positive constants. Therefore, letting $\phi=\phi_{k}^{r}, k \in \mathbf{N}$ and then adding on index $k \in \mathbf{N}$, we immediately deduce by (3.1) and (3.3) that

$$
\begin{aligned}
E\left(\sup _{0 \leq s \leq T}\left\|X_{s}\right\|_{-r}^{2}\right) \\
\leq E\left\|X_{0}\right\|_{-r}^{2}+\int_{0}^{T} \gamma(s) e^{-\mu s} d s \\
\quad+\frac{1}{2} E\left(\sup _{0 \leq s \leq T}\left\|X_{s}\right\|_{-r}^{2}\right)+K_{2} \int_{0}^{T} E\left\|B\left(s, X_{s}\right)\right\|_{L_{(2)}\left(H_{Q}, \Phi_{-r}\right)}^{2} d s
\end{aligned}
$$

Now we can easily obtain our claim by (3.1) and (3.7).

Step 3. Now we are in a position to prove our main results. Firstly, by virtue of the coercivity condition (3.1) and a similar argument to (3.13), we can get for any $\phi \in \Phi$ with $\|\phi\|_{r}=1$, 


$$
\begin{aligned}
& \sup _{t \in[N, N+1]} X_{t}[\phi]^{2} \\
& \leq X_{N}[\phi]^{2}+\int_{N}^{N+1} \gamma(s) e^{-\mu s} d s \\
& \quad+2\left[\sup _{t \in[N, N+1]}\left|\sum_{j} \int_{N}^{t} X_{s}[\phi]\left\langle B\left(s, X_{s}\right)^{\prime} \phi, v_{j}\right\rangle_{H_{Q}} d W_{s}\left[\imath^{-1} v_{j}\right]\right|\right]
\end{aligned}
$$

where $N$ is a natural number. In particular, letting $N>0$ large enough, we have for any $\epsilon_{N}>0$,

$$
\begin{aligned}
& P\left\{\sup _{t \in[N, N+1]} X_{t}[\phi]^{2} \geq \epsilon_{N}^{2}\right\} \\
& \leq \epsilon_{N}^{-2} E\left[\sup _{t \in[N, N+1]} X_{t}[\phi]^{2}\right] \\
& \leq \epsilon_{N}^{-2}\left\{E X_{N}[\phi]^{2}+\int_{N}^{N+1} \gamma(s) e^{-\mu s} d s\right. \\
& \left.\quad+2 E\left[\sup _{t \in[N, N+1]}\left|\sum_{j} \int_{N}^{t} X_{s}[\phi]\left\langle B\left(s, X_{s}\right)^{\prime} \phi, v_{j}\right\rangle_{H_{Q}} d W_{s}\left[\imath^{-1} v_{j}\right]\right|\right]\right\}
\end{aligned}
$$

which, by a similar argument to (3.14) and using (3.16), immediately deduces there exists a constant $K_{1}>0$ such that

$$
\begin{aligned}
P\left\{\sup _{t \in[N, N+1]} X_{t}[\phi]^{2} \geq \epsilon_{N}^{2}\right\} \leq & K_{1} \epsilon_{N}^{-2}\left[E X_{N}[\phi]^{2}+\int_{N}^{N+1} \gamma(s) e^{-\mu s} d s\right. \\
& \left.+\int_{N}^{N+1} E\left\|B\left(s, X_{s}\right)^{\prime} \phi\right\|_{H_{Q}}^{2} d s\right] .
\end{aligned}
$$

Therefore, by using (3.3) and (3.12), we can deduce that there exists a constant $K_{2}>0$ such that

$$
E X_{N}[\phi]^{2}+\int_{N}^{N+1} \gamma(s) e^{-\mu s} d s+\int_{N}^{N+1} E\left\|B\left(s, X_{s}\right)^{\prime} \phi\right\|_{H_{Q}}^{2} d s \leq K_{2} \cdot e^{-\tau N / 2},
$$

which, letting $\epsilon_{N}^{2}=e^{-\tau N / 4}$, immediately implies there exists a constant $K_{3}>0$ such that

$$
P\left\{\sup _{t \in[N, N+1]} X_{t}[\phi]^{2} \geq \epsilon_{N}^{2}\right\} \leq K_{3} \cdot e^{-\tau N / 4} .
$$

Finally, a Borel-Cantelli's lemma type argument completes the proof. 


\section{§4. An Example}

In this section, we shall investigate a semilinear stochastic differential equation driven by nuclear space-valued Wiener processes to illustrate the results derived in the last section.

Example 4.1. [11] White noise current injection at a point $(d>1)$. Let $L=-\Delta+I$ be an operator on $H$, where

$$
H=\left\{h \in L^{2}(\mathcal{X}, d x):\left.\frac{\partial h}{\partial x_{i}}\right|_{x_{i}=0}=\left.\frac{\partial h}{\partial x_{i}}\right|_{x_{i}=\pi}=0\right\}, \quad i=1,2, \ldots, d
$$

and $\mathcal{X}=[0, \pi]^{d}$. Then $L$ is a nonnegative-definite and self-adjoint operator on the separable Hilbert space $H$ with discrete spectrum. Let $\lambda_{j_{1}, \ldots, j_{d}}, \phi_{j_{1}, \ldots, j_{d}}$, $j_{1}, \ldots, j_{d} \geq 0$ be the eigenvalues and eigenvectors of $L$, respectively, i.e.,

$$
\lambda_{j_{1}, \ldots, j_{d}}=j_{1}^{2}+\cdots+j_{d}^{2}+1, \quad \phi_{j_{1}, \ldots, j_{d}}(x)=\phi_{j_{1}}\left(x_{1}\right) \cdots \phi_{j_{d}}\left(x_{d}\right)
$$

and

$$
\phi_{0}\left(x_{k}\right)=\left(\frac{1}{\pi}\right)^{1 / 2}, \quad \phi_{j_{k}}\left(x_{k}\right)=\left(\frac{2}{\pi}\right)^{1 / 2} \cos \left(j_{k} x_{k}\right), \quad j_{k} \geq 1 .
$$

For $r \in \mathbf{R}$ and $h \in H$, let

$$
\|h\|_{r}^{2}:=\sum_{j_{1}, \ldots, j_{d}}\left\langle h, \phi_{j_{1}, \ldots, j_{d}}\right\rangle_{H}^{2}\left(1+\lambda_{j_{1}, \ldots, j_{d}}\right)^{2 r}
$$

and

$$
\Phi:=\left\{h \in H:\|h\|_{r}<\infty, \forall r \in \mathbf{R}\right\}
$$

where $\langle\cdot, \cdot\rangle_{H}$ is the inner product on $H$. For each $r$, let $\Phi_{r}$ be the completion of $\Phi$ with respect to the norm $\|\cdot\|_{r}$. Let $\Phi^{\prime}$ be the union of all $\Phi_{r}, r \in \mathbf{R}$. Note that $\Phi_{0}=H$. Then $\Phi$ is a countably Hilbertian space and $\Phi^{\prime}$ its dual space. Likewise, it can be easily proved that the canonical injection from $\Phi_{q}$ into $\Phi_{p}$ is Hilbert-Schmidt for some $r_{1}>0$ with $q \geq p+r_{1}$ and therefore $\Phi$ is a CHNS.

Suppose process $\xi_{t}$ is the unique solution of equation

$$
d \xi_{t}=-L^{\prime} \xi_{t} d t+\left(1+t^{2}\right) e^{-t / 2} g\left(\xi_{t}\right) d W_{t}, \quad \xi_{0}=0,
$$

where $L^{\prime}$ is the dual of $L$ and $g(\cdot): \Phi^{\prime} \rightarrow \mathbf{R}$ is a bounded, Lipschitz continuous function in a strong sense. $W_{t}$ is a $\Phi^{\prime}$-valued Wiener process with $E W_{t}[\phi]=0$ and

$$
E W_{t}[\phi] W_{s}[\psi]=(t \wedge s) \phi\left(x_{0}\right) \psi\left(x_{0}\right), \quad \phi, \psi \in \Phi
$$


$W_{t}$ is $\Phi^{\prime}$-valued in a degenerate sense for we may take $W_{t}=Z_{t} \delta_{x_{0}}$ where $Z_{t}$ is a real-valued standard Wiener process and $\delta_{x_{0}}$ is the Dirac measure at $x_{0}$.

For the equation (2.8), at the moment $A(t, v)=-L^{\prime} v$ and $B(t, v)=(1+$ $\left.t^{2}\right) e^{-t / 2} g(v) I$, where $v \in \Phi^{\prime}$ and $I$ is the identity operator from $\Phi^{\prime}$ into $\Phi^{\prime}$. For simplicity of notation we denote $\left(j_{1}, \ldots, j_{d}\right)$ by $\vec{j}$. It could be proved [11] that $\forall p \geq 0, \exists q=p+1$ such that $A$ is a continuous map from $\Phi_{-p}$ into $\Phi_{-q}$ and

$$
\|A(t, v)\|_{-q} \leq\|v\|_{-p}, \quad \forall v \in \Phi_{-p} .
$$

As

$$
\left|\phi_{\vec{j}}(x)\right| \leq\left(\frac{2}{\pi}\right)^{d / 2}, \quad \forall x \in[0, \pi]^{d}
$$

we have

$$
\left|\phi\left(x_{0}\right)\right|^{2} \leq\left(\frac{2}{\pi}\right)^{d} \sum_{\vec{j}}\left\langle\phi, \phi_{\vec{j}}\right\rangle_{H}^{2}\left(1+\lambda_{\vec{j}}\right)^{2 r_{2}} \sum_{\vec{j}}\left(1+\lambda_{\vec{j}}\right)^{-2 r_{2}} \equiv \theta\|\phi\|_{r_{2}}^{2}
$$

for $r_{2}>d / 4$. Then for $p>d / 2$, the canonical injection from $H_{Q}$ to $\Phi_{-p}$ is Hilbert-Schmidt, i.e., $B(\cdot, \cdot)$ is a continuous map from $\Phi_{-p}$ to $L_{(2)}\left(H_{Q}, \Phi_{-p}\right)$. It could also be proved that $\xi . \in C\left([0, T], \Phi_{-p}\right), \forall T \in \mathbf{R}^{+}$(cf. [11]).

Note that, for CONS $\left(\phi_{\vec{j}}\right)$ and $v \in \Phi_{-p}$

$$
\begin{aligned}
\sum_{\vec{j}}\left(1+\lambda_{\vec{j}}\right)^{-2(p+1)} & \left\langle-L^{\prime} v, \phi_{\vec{j}}\right\rangle_{H}\left\langle v, \phi_{\vec{j}}\right\rangle_{H} \\
& =\sum_{\vec{j}} \frac{-\lambda_{\vec{j}}}{1+\lambda_{\vec{j}}}\left(1+\lambda_{\vec{j}}\right)^{-2(p+1 / 2)}\left\langle v, \phi_{\vec{j}}\right\rangle_{H}^{2} \\
& \leq-1 / 2\|v\|_{-(p+1 / 2)}^{2}
\end{aligned}
$$

which immediately yields that for any $v \in \Phi_{-p}$ there exists a positive constant $M(p)>0$ such that

$$
\begin{aligned}
2\langle A(t, v), v\rangle_{-(p+1)} & +\|B(t, v)\|_{L_{(2)}\left(H_{Q}, \Phi_{-(p+1 / 2)}\right)}^{2} \\
& \leq-\|v\|_{-(p+1 / 2)}^{2}+M(p)\left(1+t^{2}\right)^{2} e^{-t} .
\end{aligned}
$$

Therefore, applying Theorem 3.2 to (4.2) immediately implies that the strong solution is exponentially stable in mean square. Moreover, there exist constants $\tau>0, C(p)>0$ such that

$$
E\left\|\xi_{t}\right\|_{-(p+1 / 2)}^{2} \leq C(p) \cdot e^{-\tau t}
$$

where $p>d / 2$. In the meantime, by Theorem 3.3 the solution is also almost surely stable. 


\section{Acknowledgements}

The authors would like to thank the anonymous referee and editor for their careful comments on this work.

\section{References}

[1] Caraballo, T. and Liu, K., On exponential stability criteria of stochastic partial differential equations, Stoch. Proc. Appl., 83 (1999), 289-301.

[2] Caraballo, T. and Real, J., On the pathwise exponential stability of non-linear stochastic partial differential equations, Stoch. Anal. Appl., 12(5) (1994), 517-525.

[3] Friedman, A., Stochastic Differential Equations and Applications, 1, 2, Academic Press, New York, 1975.

[4] Friedman, A. and Pinsky, M., Asymptotic stability and spiraling properties of stochastic equations, Trans. Amer. Math. Soc., 186 (1973), 331-358.

[5] Gel'fand, I. M. and Vilenkin, N. J., Generalized Functions, 4, Academic Press, 1964.

[6] Haussmann, U. G., Asymptotic stability of the linear Itô equation in infinite dimensional, J. Math. Anal. Appl., 65 (1978), 219-235.

[7] Ichikawa, A., Stability of semilinear stochastic evolution equations, J. Math. Anal. Appl., 90 (1982), 12-44.

[8] Kallianpur, G., Mitoma, I. and Wolpert, R. L., Diffusion equations in duals of nuclear spaces, Stochastics, 29 (1990), 1-45.

[9] Kallianpur, G. and Perez-Abreu, V., Stochastic evolution equations driven by nuclearspace-valued martingale, Applied Mathematics and Optimization, 17 (1988), 237-272.

[10] Kallianpur, G. and Wolpert, R. L., Infinite dimensional stochastic differential equation models for spatially distributed neurons, Appl. Math. Optim., 12 (1984), 125-172.

[11] Kallianpur, G. and Xiong, J., Stochastic Differential Equations in Infinite Dimensional Spaces, Lecture Notes Monograph series, Inst. Math. Statis., 26 (1995).

[12] Liu, K., On stability for a class of semilinear stochastic evolution equations, Stoch. Proc. Appl., 70 (1997), 219-241.

[13] Mao, X. R., Exponential Stability of Stochastic Differential Equations, Marcel Dekker, Inc, 1994.

[14] Da Prato, G. and Zabczyk, J., Stochastic Equations in Infinite Dimensions, Cambridge Univ. Press, 1992.

[15] Tuckwell, H., Stochastic Processes in Neurosciences, Society for Industrial and Applied Mathematics, Philadelphia, 1989.

[16] Walsh, J. B., A stochastic model of neural response, Adv. in Appl. Probab., 13 (1981), 231-281. 